\title{
Multi-sensor system for dynamic deformation and vibration measurements at high-speed rotors
}

\author{
Katrin Philipp ${ }^{1}$, Robert Kuschmierz'1, Angelos Filippatos², Albert Langkamp², Maik Gude², Andreas \\ Fischer $^{1}$, Jürgen Czarske ${ }^{1}$ \\ ${ }^{1}$ Laboratory for Measuring and Testing Techniques, Technische Universität Dresden, Germany, \\ katrin.philipp@tu-dresden.de \\ 2 Institute of Lightweight Engineering and Polymer Technology, Technische Universität Dresden,
} Germany

\begin{abstract}
:
The in-situ characterization of the material behavior of glass-fiber reinforced polymer (GFRP) disc rotors under dynamic load is an important step towards the development of novel, lightweight materials for several applications, e.g. the aerospace industry. However, the dynamic rotor behavior is complex and difficult to determine. In order to develop improved models and simulation techniques for the optimization of composite materials, the deformation of rotors under dynamic load has to be investigated. A four component multi-sensor system is applied for in-situ monitoring of the dynamic rotor expansion. A novel signal processing algorithm is used to take tumbling motion into account, which previously restricted the precision of radial expansion measurements. As a result, micron precision for the angular resolved rotor expansion is achieved at temporal resolutions of only one rotor revolution, corresponding to an increased precision of the rotor expansion by a factor of three to six over the state-of-the-art approach. Eventually the mean rotor expansion in dependency of the rotational speed is determined with submicron precision.
\end{abstract}

Key words: multi-sensor system, dynamic deformation monitoring, high-speed composite rotors, signal processing, optical metrology.

\section{Motivation}

The in-situ characterization of the material behavior of glass-fiber reinforced polymer (GFRP) disc rotors under dynamic load is an important step towards the development of novel, lightweight materials for several applications, e.g. the aerospace industry [1]. Monitoring the dynamic rotor deformation due to the centrifugal forces reveals information about damage initiation and development within the rotor volume [2]. This eventually leads to improved models of the material behavior and might allow predicting final rotor failure. However, typical rotor diameters of about $50 \mathrm{~cm}$ and radial expansions in the range of only a few $100 \mu \mathrm{m}$ complicate the measurement of the dynamic rotor deformation.

The application of strain gauges enables local deformation measurements [3]. However, they can rip of due to the high rotational speeds, if they are attached on the rotor surface. Integrated strain gauges are invasive and may change the rotor behavior under dynamic load. Electrical sensors like capacitive, inductive or eddy current probes are well-established for measurements at metallic rotors, but the low magnetic permeability and electrical conductivity restricts the applicability at composite rotors [4]. Placing several optical distance sensors around the circumference of the rotor enables the contactless, destruction-free measurement of the radial expansion [5]. In contrast to optical sensors like triangulation [6], chromatic confocal sensing [7] and optical coherence tomography [8], laser Doppler distance sensors are suitable for measurements at high surface velocities and translucent materials [9]. However, the precision of previous measurements of the radial expansion using three laser Doppler distance sensors is limited to about $30 \mu \mathrm{m}$ at temporal resolutions of one rotor revolution [2]. Since the laser Doppler distance sensor has been optimized for the application at the investigated rotor material, the measurement precision is

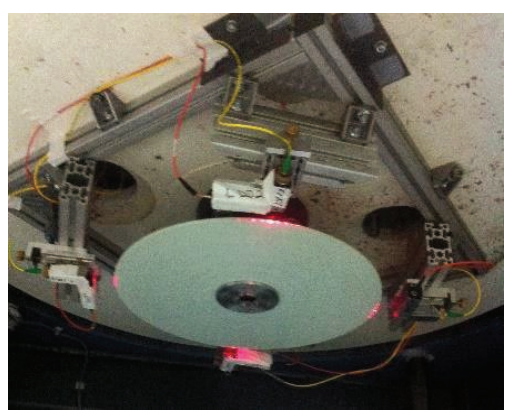

Fig. 1. Multi-sensor system applied at a glass-fiber reinforced polymer disc rotor. 
mainly limited by the signal processing. In particular, tumbling motions with amplitudes up to $150 \mu \mathrm{m}$ at the used test rig [5] were not taken into account in previous measurements [2] and are the main restriction of achieved measurement precision.

The aim of this contribution is the application of a four-component multi-sensor system (Fig. 1) for radial expansion measurements at glassfiber reinforced polymer rotors with micron measurement precision. For this purpose, a novel signal processing algorithm is introduced, that takes into account the tumbling motion of the rotor. The multi-sensor system is eventually applied for rotor expansion measurements with rotational speeds from $600 \mathrm{rpm}$ to $7200 \mathrm{rpm}$. The mean rotor expansion is determined with submicron measurement precision.

\section{Multi-sensor system}

The multi-sensor system consists of four distance sensors equally distributed along the circumference of the rotor (Fig. 2).

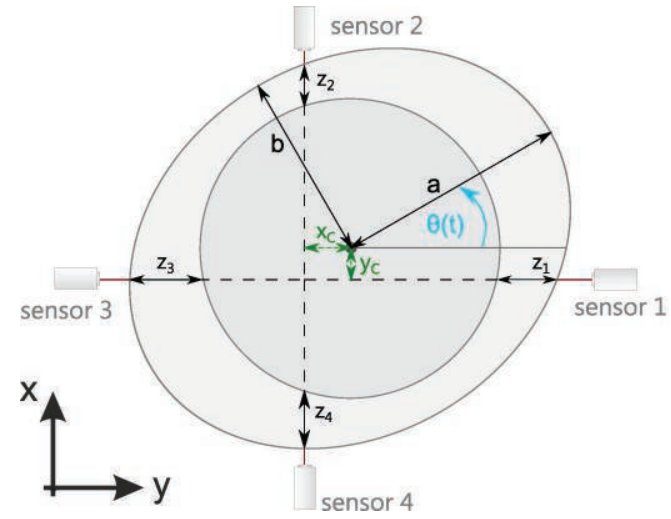

Fig. 2. Setup of multi-sensor system consisting of four sensors equally distributed along the circumference of the rotor.

The sensor shape is defined by the rotor radius $r=r_{0}+r_{\Delta}$, that consists of the initial radius $r_{0}$ of the rotor at rest and the radius expansion $r_{\Delta}$ (Fig. 2 ). Note that the time dependency of the variables is not explicitly denoted for better readability. The tumbling motion is described by the rotor center position $\left(x_{c}, y_{c}\right)$ and the current rotor orientation by $\theta=\omega t$. The corresponding rotational frequency $\omega$ is obtained by an inductive rotational speed sensor. The sensor signals $z_{i}, i=1,2,3,4$ specify the distance between the rotor surface position at the beginning of the measurement and current rotor surface. Thus, the sensor signals contain a superposition of radial expansion and tumbling motion of the rotor. The simultaneous determination of radial expansion and rotor center position is the measurement task to be solved by signal processing algorithms.
The multi-sensor system is realized with laser Doppler distance sensors, since these sensors have already been successfully applied at high surface speeds up to $600 \mathrm{~m} / \mathrm{s}$ [2] and at translucent materials [9].

\section{Signal processing}

The aim of the signal processing algorithm is the simultaneous determination of the radial rotor expansion $r_{\Delta}(\vartheta, t)$ and the rotor center position $\left(x_{c}, y_{c}\right)$. At a given time, the four sensors deliver the sensor distance signals $\mathrm{z}_{\mathrm{i}}(\mathrm{t}), i=$ $1,2,3,4$. The current rotor orientation $\theta(t)=\omega \cdot t$ is determined using an inductive rotational speed sensor.

The assuming of small tumbling motion amplitudes yields the simplified equation system

$$
z_{i}=r_{\Delta}\left(\phi_{i}-\theta\right)+x_{c} \cos \left(\phi_{i}\right)+y_{c} \sin \left(\phi_{i}\right),
$$

with the angular sensor positions $\phi_{i}=(i-1) \cdot \frac{\pi}{2}$. The equation system resulting from $\mathrm{Eq}$. (1) at a given measurement time $t$ is underdetermined, since it consists of four equations and 12 parameters. Thus, additional assumptions have to be made.

The state-of-the-art approach [2] assumes zeroaverage tumbling motion and applies temporal averaging to Eq. (1) over integer multiples of the rotor revolution time $T_{\text {revolution }}=\frac{2 \pi}{\omega}$. In order to combine the information of all sensors at a given rotor angle $\vartheta$, the sensor signals are time-shifted

$$
\tilde{z}_{i}(t)=z_{i}\left(t+\frac{\phi_{i}}{2 \pi} \cdot T_{\text {revolution }}\right) .
$$

The radial expansion is then determined by averaging over all four sensor signals and over all measured points, i.e.

$$
r_{\Delta}(\vartheta)=\frac{1}{4 N_{r}} \sum_{i=1}^{4} \sum_{n_{r}=0}^{N_{r}-1} \tilde{z}_{i}\left(n_{r} \cdot T_{\text {revolution }}\right) \text {. }
$$

If the tumbling motion is zero-average, i.e. $\overline{x_{c}(t)}=\overline{y(t)}=0$, the tumbling term of Eq. (1) is eliminated by the temporal averaging in Eq. (3). Consequently, the state-of-the-art approach enables the determination of arbitrary (convex) rotor expansions at the cost of temporal resolution. If the tumbling motion is not zeroaverage as at the used test rig [5], the measured rotor expansion is distorted, leading to increased measurement uncertainties.

In order to eliminate the influence of the tumbling motion on the measurement precision, a novel signal processing algorithm is introduced. As shown in previous experiments, the expansion of the investigated rotor type is elliptical [2]. Exploiting this knowledge allows solving 


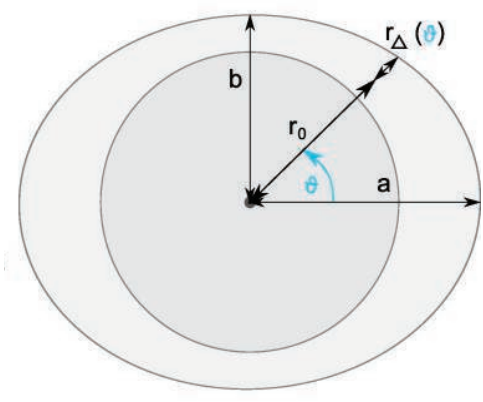

Fig. 3. Illustration of the radial expansion $\mathrm{r}_{\Delta}(\vartheta)$ with major and minor axis $a$ and $b$ of ellipse, respectively.

equation system (1) unambiguously. The radius of an ellipse equals

$$
r_{\text {ellipse }}(\vartheta)=\frac{a b}{\sqrt{a^{2} \sin ^{2} \vartheta+b^{2} \cos ^{2} \vartheta}}
$$

with angular rotor position $\vartheta$, the major and minor axes of the ellipse $a$ and b, respectively (Fig. 3).

Inserting $r_{\Delta}=r_{\text {ellipse }}-r_{0}$ into Eq. (1) yields the solution

$$
\begin{gathered}
a=\frac{A}{2} \sqrt{\cos ^{2} \omega t+\sin ^{2} \omega t \frac{A^{2} \cos ^{2} \omega t-B^{2} \sin ^{2} \omega t}{B^{2} \cos ^{2} \omega t-A^{2} \sin ^{2} \omega t}} \\
b=\frac{B}{2} \sqrt{\cos ^{2} \omega t+\sin ^{2} \omega t \frac{A^{2} \cos ^{2} \omega t-B^{2} \sin ^{2} \omega t}{B^{2} \cos ^{2} \omega t-A^{2} \sin ^{2} \omega t}} \\
x_{c}=\frac{1}{2}\left(z_{1}-z_{3}\right) \\
y_{c}=\frac{1}{2}\left(z_{2}-z_{4}\right)
\end{gathered}
$$

with $A=2 r_{0}+z_{1}+z_{2}$ and $B=2 r_{0}+z_{2}+z_{4}$. Thus, this novel signal processing algorithm enables the separation and simultaneous determination of rotor expansion and tumbling motion and consequently an increased measurement precision of the rotor expansion.

\section{Validation of the measurement system}

The multi-sensor system is applied at glass-fiber reinforced polymer disc rotors with rotational speeds of up to $7200 \mathrm{rpm}$. The reinforcement of the investigated rotor consist of fibers orientated in $0^{\circ}, 45^{\circ}, 90^{\circ}$ and $-45^{\circ}$ direction. The mass percentage of the fibers varies with the orientation, as listed in Table 1. The complete rotor properties are described in [10].

Tab. 1: Properties of rotor glass fiber reinforcement.

\begin{tabular}{|c|c|c|}
\hline orientation & $\begin{array}{c}\text { Filament type } \\
\text { (GF-Rowing) }\end{array}$ & $\begin{array}{c}\text { Mass } \\
\text { percentage }\end{array}$ \\
\hline $0^{\circ}$ & $2400 / 1200$ tex & $48.7 \%$ \\
\hline $45^{\circ}$ & 300 tex & $23 \%$ \\
\hline $90^{\circ}$ & 200 tex & $4.8 \%$ \\
\hline$-45^{\circ}$ & 300 tex & $23 \%$ \\
\hline
\end{tabular}

The selected layup and the resulting in plane anisotropic material cause elliptic rotor expansion under dynamic load. Using the stateof-the-art signal processing algorithm, the elliptic form of the expansion is confirmed (Fig. 4).

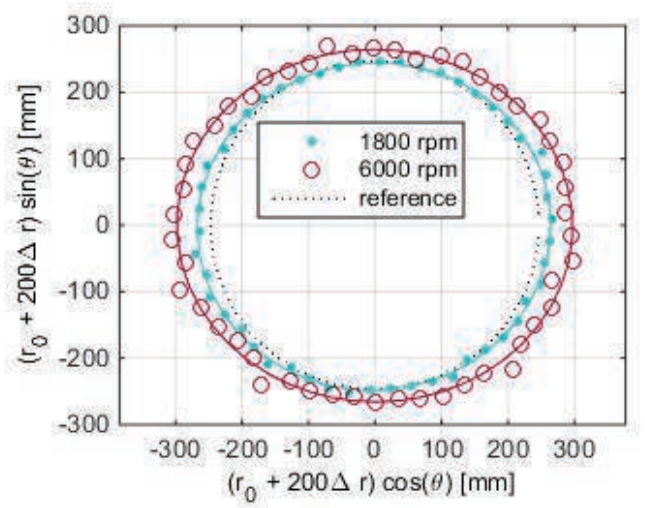

Fig. 4. Angular resolved rotor expansion determined by state-of-the-art approach and corresponding elliptic fits (solid lines).

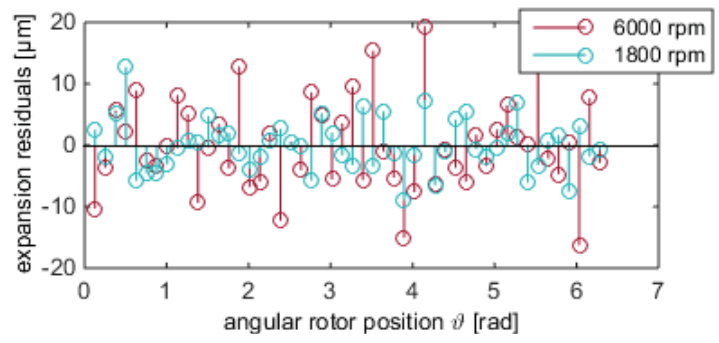

Fig. 5. Residuals of measured rotor expansion and elliptic fit.

The residuals are stochastically distributed (Fig. 5 ) and thus justify the application of the presented signal processing algorithm. As a consequence, the tumbling motion of the rotor is resolved with a measurement rate of $3 \mathrm{kHz}$ (Figs. 6 and 7$)$.
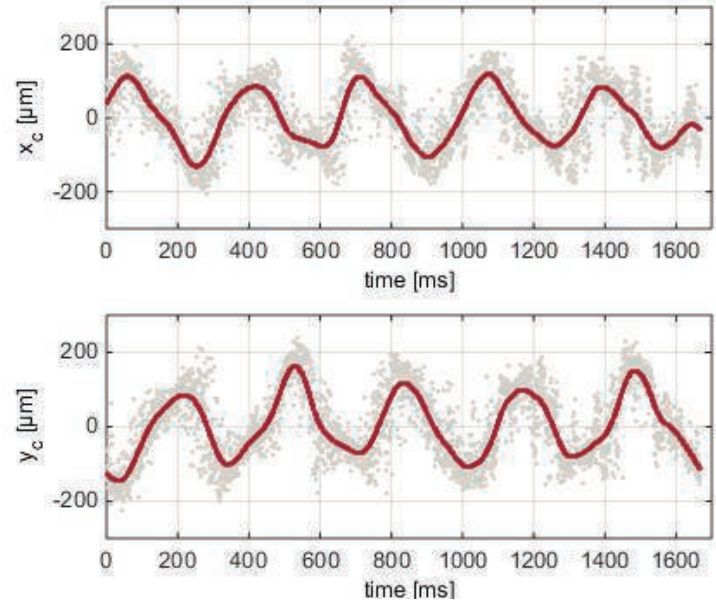

Fig. 6. Tumbling motion described by rotor center coordinates $x_{c}(t)$ and $y_{c}(t)$ at a rotational speed of $1800 \mathrm{rpm}$. Gray points are single point measuremens and solid lines are correspondina rearession curves. 


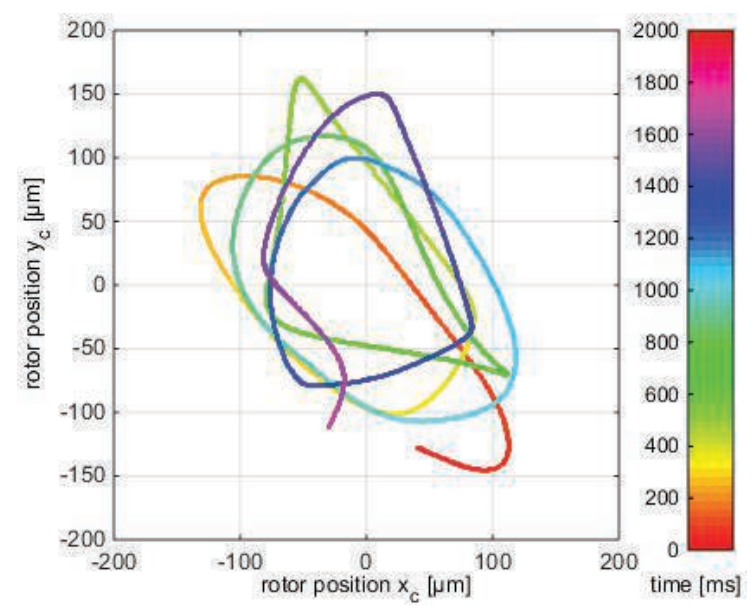

Fig. 7. Map of unsteady rotor tumbling motion reconstructed out of regression curves in Fig. 6.

The mean tumbling amplitude

$$
A_{\text {tumbling }}=\frac{1}{N} \sum_{n=1}^{N} \sqrt{x_{c}^{2}\left(t_{n}\right)+y_{c}^{2}\left(t_{n}\right)}
$$

is determined by averaging over all measurements $n=1,2, \ldots, N$ for all investigated rotational speeds. Since the state-of-the-art approach requires measurement times of at least one full rotor revolution, both measurements are conducted over one revolution. The measurement uncertainty $\sigma_{r_{\Delta}}$ using the proposed signal processing algorithm does not depend on the tumbling amplitude $A_{\text {tumbling }}$ in contrast to the state-of-the-art approach (Fig. 8). This yields to an increase of the measurement precision by a factor of three to six, depending on the tumbling amplitude. This improved precision is achieved because the novel algorithm takes into account the tumbling

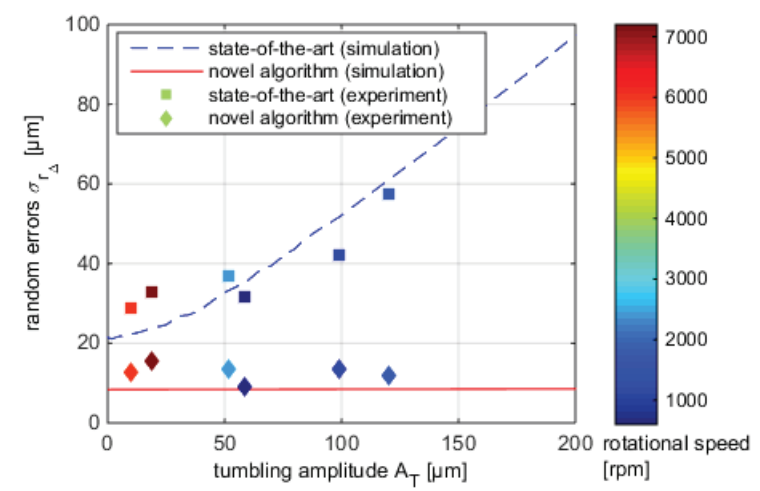

Fig. 8. Uncertainties of angular resolved rotor expansion for measurement during one rotor revolution.

motion.

Simulations using generated signals with equal parameters as in the measurements are in agreement with the measurement results obtained by the sensors (Fig. 8). The uncertainty for measurement duration of $N$ rotor revolutions is further reduced to $\frac{t \sigma_{r_{\Delta}}}{\sqrt{N}}$ with the factor $t$ of the corresponding Student's t-distribution. The rotor expansion measurements are conducted over 40 rotor revolutions, resulting in $3 \mu \mathrm{m}$ precision of the angular resolved rotor expansion. The mean radial expansion increases quadratic with the rotational speed due to centrifugal forces following Hooke's law (Fig. 9). The standard

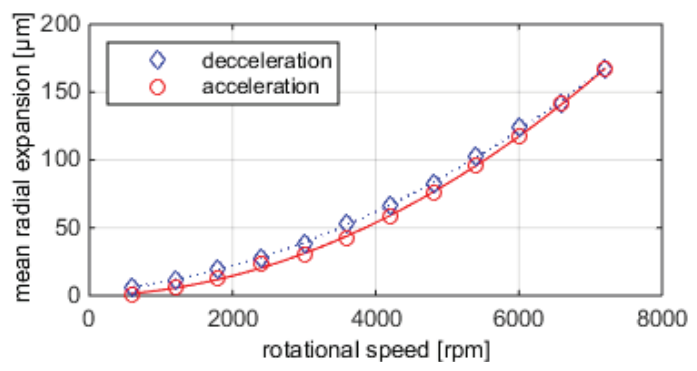

Fig. 9. Mean radial rotor expansion with increasing and decreasing rotational speeds, respectively.

deviation between measured expansion and quadratic fit is only $850 \mathrm{~nm}$.

\section{Outlook: Out-of-plane vibration monitoring}

The sensor system is also adaptable for out-ofplane vibration measurements by locating the sensors parallel to the rotation axis of the rotor, as illustrated in Fig. 10.

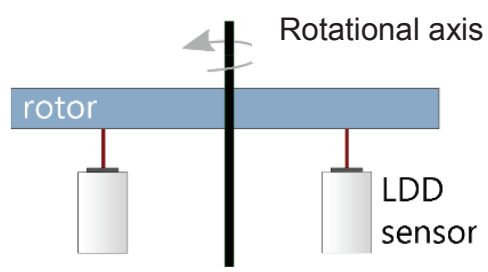

Fig. 10. Adaption of multi-sensor system for out-ofplane vibration measurements.

As a proof-of-concept, the adapted sensor system is applied at a rotor with rotational speed of $1850 \mathrm{rpm}$. This corresponds to the first eigenfrequency of the rotor, aiming at initiating out-of-plane vibrations. The spectrum of the measured vibration is obtained by a fourier transformation of the measured sensor distance signals. The spectrum has peaks at first eigenfrequency and its harmonics (Fig. 11).

Consequently, these first measurements suggest, that the adapted multi-sensor system is a promising tool for out-of-plane vibration measurement. 


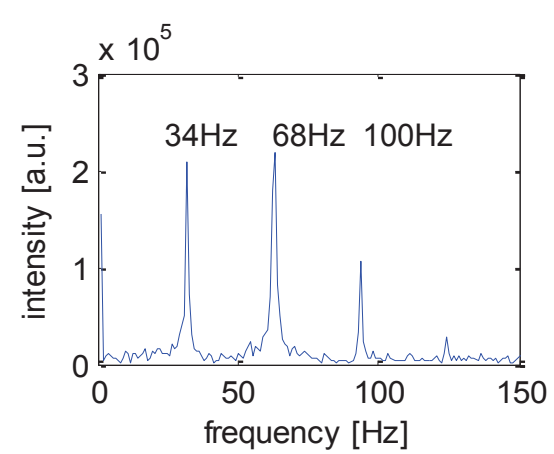

Fig. 11. Spectrum of measured out-of-planevibrations with peaks at first eigenfrequency and corresponding harmonics.

\section{Conclusion}

Exploiting the knowledge of the elliptic rotor shape enables the simultaneous determination of the rotor expansion and the tumbling motion with measurement rates of $3 \mathrm{kHz}$. Micron measurement precision of the angular resolved rotor expansion is achieved at temporal revolutions corresponding to one rotor revolution. This correlates to an improvement of the expansion precision by a factor three to six compared to the state-of-the-art approach (depending on the tumbling amplitude). The mean expansion of the rotor is obtained with sub-micron precision for measurement times of $40 \cdot T_{\text {revolution }}$. In conclusion, the proposed multisensor system is an appropriate tool for the characterization of fiber-reinforced composite rotors.

\section{References}

[1] C. Bakis, L. Bank, V. Brown, E. Cosenza, J. Davalos, J. Lesko, A. Machida, S. Rizkalla, and T. Triantafillou, "Fiber-Reinforced Polymer Composites for ConstructionState-of-the-Art Review," J. Compos. Constr., vol. 6, no. 2, pp. 73-87, 2002.

[2] R. Kuschmierz, A. Filippatos, P. Günther, A Langkamp, W. Hufenbach, J. Czarske, and A. Fischer, "In-process, non-destructive, dynamic testing of high-speed polymer composite rotors," Mech. Syst. Signal Process., vol. 54-55, pp. 325-335, Mar. 2015.

[3] F. Gasco, P. Feraboli, J. Braun, J. Smith, P. Stickler, and L. DeOto, "Wireless strain measurement for structural testing and health monitoring of carbon fiber composites," Compos. Part A Appl. Sci. Manuf., vol. 42, no. 9, pp. 1263-1274, 2011.

[4] D. A. Subramani, V. Ramamurti, and K. Sridhara, "Numerical analysis and experimental verification of the radial growth of a turbocharger centrifugal compressor impeller," J. Strain Anal. Eng. Des., vol. 32, no. 2, pp. 119-128, 1997.

[5] P. Günther, F. Dreier, T. Pfister, J. Czarske, T. Haupt, and W. Hufenbach, "Measurement of radial expansion and tumbling motion of a high-speed rotor using an optical sensor system," Mech. Syst. Signal Process., vol. 25, no. 1, pp. 319-330, 2011.

[6] R. G. Dorsch, G. Häusler, and J. M. Herrmann, "Laser triangulation: fundamental uncertainty in distance measurement," Appl. Opt., vol. 33, no. 7, pp. 1306-1314, 1994.

[7] A. K. Ruprecht, K. Koerner, T. F Wiesendanger, H. J. Tiziani, and W. Osten, "Chromatic confocal detection for high-speed microtopography measurements," Proc. SPIE, vol. 5302. pp. 53-60, 2004.

[8] D. Markl, G. Hannesschläger, A. Buchsbaum, S. Sacher, J. G. Khinast, and M. Leitner, "In-line quality control of moving objects by means of spectral-domain OCT," Opt. Lasers Eng., vol. 59, pp. 1-10, Aug. 2014.

[9] K. Philipp, N. Koukourakis, R. Kuschmierz, C. Leithold, A. Fischer, and J. Czarske, "Optical dynamic deformation measurements at translucent materials," Opt. Lett., vol. 40, no. 4, 2015.

[10] M. Gude, A. Filippatos, A. Langkamp, W. Hufenbach, R. Kuschmierz, A. Fischer, and J. Czarske, "Model assessment of a composite mock-up bladed rotor based on its vibration response and radial expansion," Compos. Struct., vol. 124, pp. 394-401, Jun. 2015. 